\title{
Hydrothorax with alveolar-pleural fistula mimicking re-expansion pulmonary edema during liver transplantation
} -a case report-

\author{
Jae Hee Woo, Rack Kyung Chung, Hee Jung Baik, and Youn Jin Kim \\ Department of Anesthesiology and Pain Medicine, Ewha Womans University School of Medicine, Seoul, Korea
}

\begin{abstract}
We present a case of an alveolar-pleural fistula with hepatic hydrothorax in a patient undergoing orthotropic liver transplantation, which was detected by drainage of transudate through an endotracheal tube during operation. A standard endotracheal tube was changed to a double-lumen tube to provide differential lung ventilation. The patient was diagnosed with an alveolar-pleural fistula by direct vision of an air leak during positive-pressure ventilation through a diaphragmatic incision. There was still a concern about worsening his ventilation due to persistent aspiration of pleural effusion towards the ipsilateral lung during the remaining operation period. Surgeon repaired the defect on the exposed lung surface via diaphragmatic opening. Anesthesiologists should consider an alveolar-pleural fistula as a possible differential diagnosis with re-expansion pulmonary edema when transudate emanating from the endotracheal tube is obtained in patients with massive hydrothorax.
\end{abstract}

Key Words: Fistula, Hydrothorax, Liver transplantation.

Hepatic hydrothorax is defined as significant pleural effusion and is one of the complications of end-stage liver disease, with a prevalence of $5-10 \%$ among cirrhotic patients [1]. Its ultimate treatment is orthotropic liver transplantation (OLT). Thoracen-

Corresponding author: Rack Kyung Chung, M.D., Ph.D.

Department of Anesthesiology and Pain Medicine, Ewha Womans University School of Medicine, 1071, Anyangcheon-ro, Yangcheon-gu, Seoul 158-710, Korea

Tel: 82-2-2650-5039, Fax: 82-2-2655-2924

E-mail: rkchung@ewha.ac.kr

Received: October 27, 2014.

Revised: 1st, December 15, 2014; 2nd, December 18, 2014.

Accepted: December 21, 2014.

This article includes supplemental video clips.

Korean J Anesthesiol 2015 April 68(2): 184-187

http://dx.doi.org/10.4097/kjae.2015.68.2.184 tesis may cause serious complications, such as pneumothorax, air embolism, and re-expansion pulmonary edema (RPE), and is not usually recommended as a maintenance treatment for hydrothorax in patients undergoing OLT. However, it is still the most effective method of resolving massive pleural effusion to facilitate intraoperative patient ventilation [2]. While foamy and bloody fluid drainage from an endotracheal (ET) tube during general anesthesia commonly suggests RPE, clear fluid drainage through the ET tube can develop when pleural effusion is superimposed on a pre-existing fistula between the airway and pleura.

To our knowledge, this is the first reported case of an alveolar-pleural fistula (APF) in a patient undergoing OLT, which was detected by the drainage of clear-yellowish fluid through an ET tube, diagnosed by direct vision of an air leak through a diaphragmatic incision and successfully managed with prompt suction, a lung-isolation strategy, and surgical repair of the fistula during the operation.

(c) This is an open-access article distributed under the terms of the Creative Commons Attribution Non-Commercial License (http://creativecommons.org/ licenses/by-nc/3.0/), which permits unrestricted non-commercial use, distribution, and reproduction in any medium, provided the original work is properly cited. 


\section{Case Report}

A 55-year-old, $167 \mathrm{~cm}, 70 \mathrm{~kg}$ male suffering from alcoholic end-stage liver cirrhosis with a 'model for end stage liver disease' score of 15 points, and Child-Pugh class C, was scheduled for deceased-donor liver transplantation. He had a history of hepatic encephalopathy, refractory ascites, and undergoing a repetitive paracentesis over the previous 6 months. When he first arrived at our hospital 3 months earlier, he had suffered from hepatic hydrothorax, which waxed and waned. Pleural fluid analysis revealed transudative fluid. The preoperative transthoracic echocardiography revealed elevated right ventricular systolic pressure $(46 \mathrm{mmHg}$ ) without coexisting cardiopulmonary disease. The preoperative chest computed tomography showed massive pleural effusion and related passive atelectasis in the right middle and lower lobe (Fig. 1). On the day of surgery, he was in mild respiratory distress and a plain chest radiograph revealed massive pleural effusion of the right lung and associated lung collapse (Fig. 2). An 8.5-French percutaneous pigtail catheter was placed in the right pleural space $3 \mathrm{~h}$ before surgery and $600 \mathrm{ml}$ of transudative pleural fluid were drained naturally.

Anesthesia was induced with fentanyl $(100 \mu \mathrm{g})$, midazolam (3 mg), thiopental sodium (250 mg), and rocuronium (40 mg).

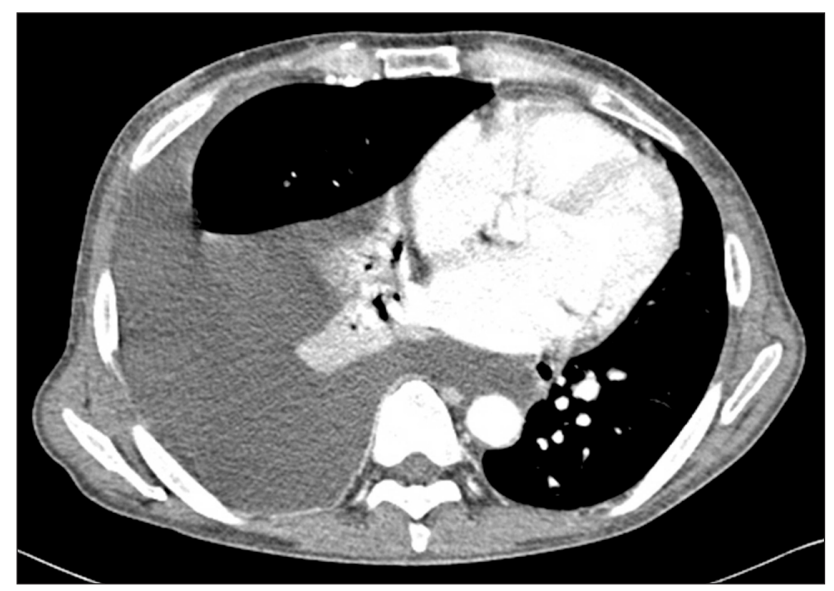

Fig. 1. Preoperative chest computed tomography shows massive pleural effusion and passive atelectasis in the right middle and lower lobe.
Anesthesia was maintained with a 50\% oxygen/air mixture and sevoflurane. Neuromuscular block was maintained with cisatracurium. Hemodynamic monitoring included the right radial and femoral arterial, central venous, pulmonary arterial, and pulmonary capillary wedge pressures, and cardiac output measurements (Vigilance monitoring, Edwards Lifesciences, Irvine, CA, USA) using pulmonary artery catheter (CCOmbo V; 7.5 French, Edwards Lifesciences).

The prehepatic and anhepatic phase was uneventful and during that period, $500 \mathrm{ml}$ of transudate were drained through the pigtail catheter. After reperfusion of a graft, he required a bolus of epinephrine $(10 \mu \mathrm{g})$, dobutamine infusion $(5-10 \mu \mathrm{g} / \mathrm{kg} / \mathrm{min})$ and norepinephrine infusion $(0.05-0.1 \mu \mathrm{g} / \mathrm{kg} / \mathrm{min})$ to maintain mean arterial pressure $>65 \mathrm{mmHg}$. At this time, his ventilation was well controlled and the results of arterial blood gas analysis were unremarkable, as shown in Table 1. However, $30 \mathrm{~min}$ after reperfusion, we found a straw-colored fluid emanating from the ET tube. The trachea was intermittently suctioned and total volume suctioned via ET tube was over $500 \mathrm{ml}$ in $30 \mathrm{~min}$. The characteristics of the regurgitated fluid were similar to those of fluid

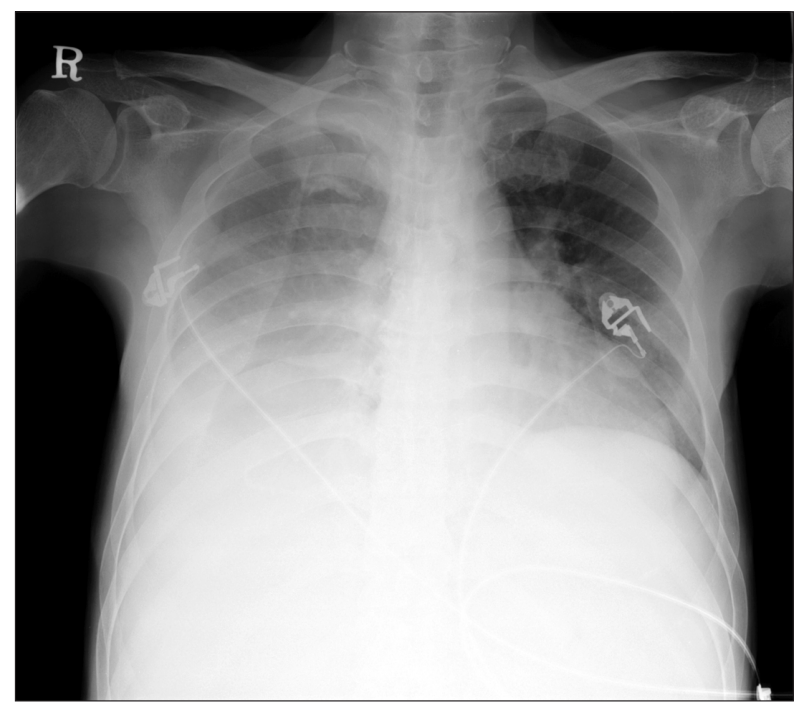

Fig. 2. Preoperative chest radiograph reveals massive pleural effusion of the right lung.

Table 1. Mechanical Ventilation and Arterial Blood Gas Analysis during Perioperative Period

\begin{tabular}{lccccrr}
\hline & $\mathrm{FIO}_{2}$ & $\mathrm{pH}$ & $\mathrm{PaCO}_{2}(\mathrm{mmHg})$ & $\mathrm{PaO}_{2}(\mathrm{mmHg})$ & $\mathrm{BE}(\mathrm{mmol} / \mathrm{L})$ & $\mathrm{SaO}(\%)$ \\
\hline Baseline & Room air & 7.43 & 33 & 83.1 & -2.4 & 97 \\
Prehepatic phase & 0.5 & 7.46 & 35 & 242 & 1.1 & 100 \\
Anhepatic phase, 40 min & 0.5 & 7.31 & 38 & 204 & -7.2 & 100 \\
Post-reperfusion, 5 min & 0.5 & 7.20 & 46 & 215 & -6.5 & 100 \\
Post-reperfusion, 40 min & 0.5 & 7.28 & 40 & 256 & -4.0 & 100 \\
Reperfusion, 2 h & 0.5 & 7.31 & 44 & 188 & -6.2 & 100 \\
Abdominal closure & 0.5 & 7.27 & 45 & 100 & \\
\hline
\end{tabular}


drained through the pigtail catheter, which decreased visibly at that time. Hypoxemia did not occur with a $\mathrm{FIO}_{2}$ of 0.5 , as indicated by the stable $\mathrm{SpO}_{2}$ during the entire intraoperative period. Thus, we suspected the presence of a connection between the airway and pleural space on the affected side and that the pleural fluid on the right side was regurgitated towards the ET tube. We changed a standard ET tube to a 37-French double-lumen tube (DLT; Covidien, Mansfield, MA, USA) to provide differential lung ventilation. A regurgitated fluid was obtained continuously from the right bronchus, but not from the left bronchus. After a discussion with the operating surgeons and a thoracic surgeon, we decided to open the diaphragm to check for the presence of

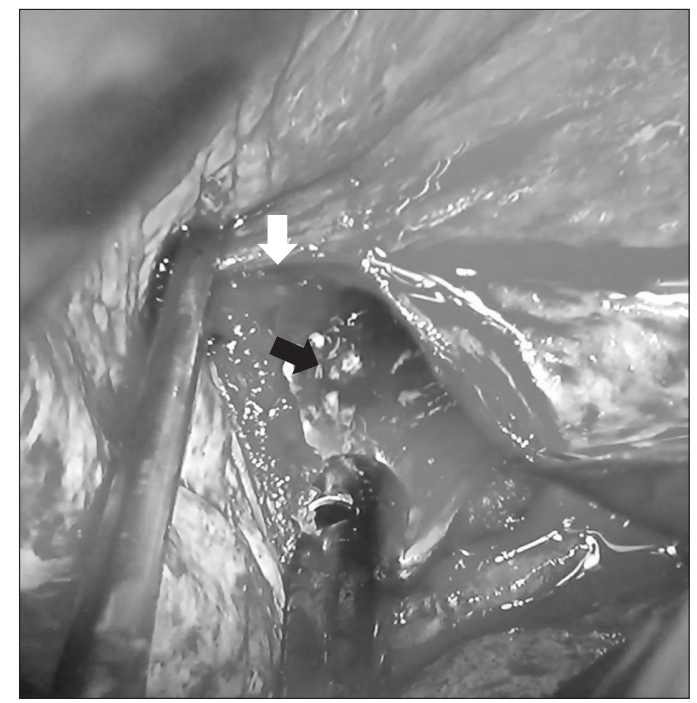

Fig. 3. The operation field view shows an air leakage (black arrow) via a $1.0 \mathrm{~cm}$-defect on the right lung surface during positive-pressure ventilation, which is exposed through a $1.5 \mathrm{~cm}$-incision of the right side of the diaphragm (white arrow) (Video 1).
APF. Air leakage via a $1.0 \mathrm{~cm}$ defect on the right lung surface during positive-pressure ventilation was detected through a 1.5 $\mathrm{cm}$ incision of the right side of the diaphragm (Fig. 3 and Video 1). The APF and incised diaphragm was repaired with a suture with a minimal residual APF. The remaining surgical procedure was otherwise uneventful. At the end of surgery, the chest tube was inserted in the right pleural cavity. During the 550-min anesthesia, 7,400 $\mathrm{ml}$ of crystalloid, $200 \mathrm{ml}$ of $20 \%$ albumin, 12 units of leukocyte depleted red blood cell, and 10 units of fresh frozen plasma were infused. Urine output was $880 \mathrm{ml}$ and the estimated blood loss was about 10,000 $\mathrm{ml}$.

Following the surgery, the patient was transferred to the intensive care unit with the placement of a DLT. An immediate postoperative chest radiograph suggested a decreased pleural effusion and mild bilateral pulmonary edema (Fig. 4A). As the amount of regurgitated fluid decreased, the DLT was replaced with a standard ET tube on the day after the operation. On the second postoperative day, pneumothorax developed on the right side (Fig. 4B), which resolved 2 days later. The ET tube was removed on the fourth postoperative day. In total, $500-1,000 \mathrm{ml}$ of drainage were obtained daily via the chest tube during 2 weeks with ongoing ascites. Drainage decreased slowly thereafter, and the chest tube was removed on postoperative day 21 (Fig. 4C).

\section{Discussion}

Cirrhotic patients have an inability to maintain extracellular fluid within normal limits with a resulting accumulation of fluid in the pleural, peritoneal, and interstitial spaces [3]. Several mechanisms have been suggested to explain the development of hepatic hydrothorax. They include the passage of ascites into the pleural space through diaphragmatic defects, which is facilitated by a negative intrathoracic pressure, and the leakage of fluid into
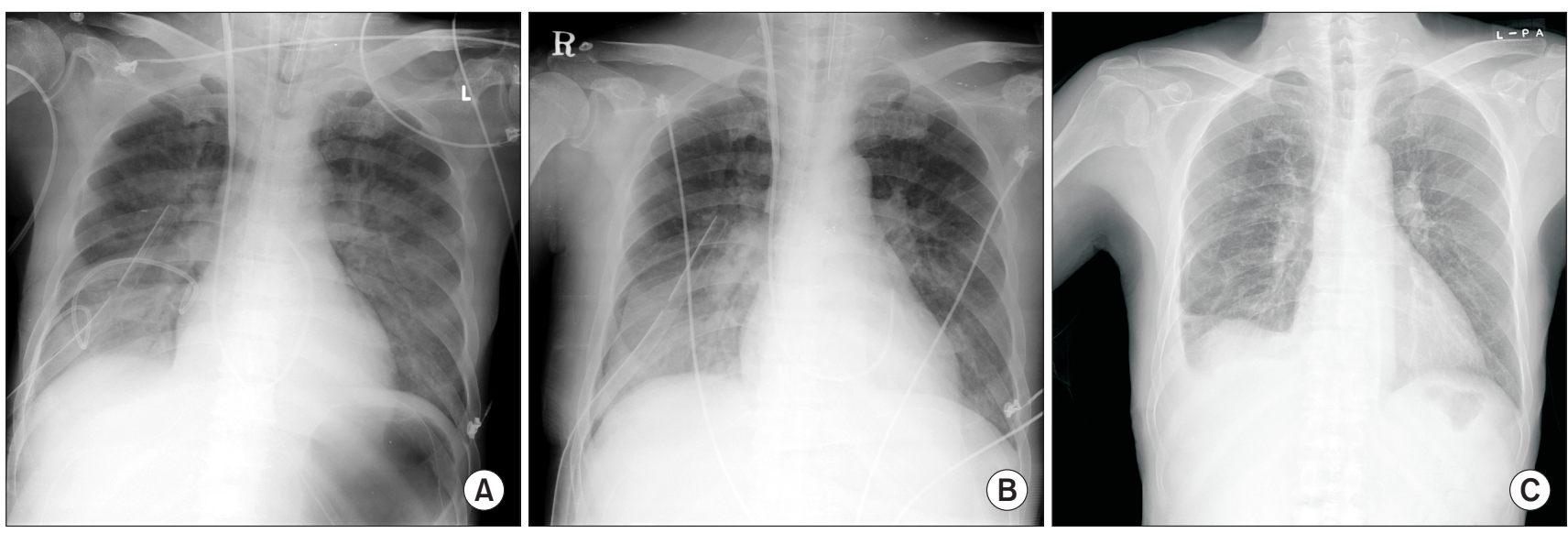

Fig. 4. (A) Immediate postoperative chest radiograph shows a decreased pleural effusion and newly appeared pulmonary edema in both lungs with right chest tube and pigtail catheter inserted. (B) A film on the second postoperative day reveals newly developed right pneumothorax. (C) A chest radiograph taken on the postoperative 21 st day shows improvement of pleural effusion, pulmonary edema and pneumothorax. 
the pleural space due to decreased colloid osmotic pressure [4]. Hepatic hydrothorax can also occur in the absence of ascites [5].

In our patient, we considered that the ongoing formation of pleural effusion spilled over through an APF, which occurred during surgery or just before surgery. APF has several possible causes, such as a complication of thoracentesis, mechanical ventilation, and pre-existing lung parenchymal disease. However, preoperative chest computed tomography showed only massive pleural effusion and related passive atelectasis in the right middle and lower lobe without other lung parenchymal diseases, such as bullae. If the patient had an APF long before undergoing OLT, he might have experienced symptoms related with an APF. Thus, we concluded that an APF occurred when performing thoracentesis, $3 \mathrm{~h}$ before the surgery, and the size of injured pleura increased with positive pressure ventilation. We considered that drainage through the ET tube did not occur at the beginning of the positive pressure ventilation for two reasons. First, the patency of the pigtail catheter might have been maintained for some time. Thereafter, it was thought that the drainage failed due to its migration, and obscuration of its lumen triggered a diversion of pleural effusion towards the ET tube. At the time at which fluid was noticed in the ET tube, the pigtail catheter might have been sealed by chest wall contact, and its drainage decreased markedly. Second, intraoperative positive pressure ventilation re-expanded the severely collapsed atelectatic lung, which could divert and shift the fluid towards the ET tube slowly.

In the case presented, RPE should be ruled out because it is a well-known complication associated with thoracentesis and the drainage of transudate via an ET tube is one of the important signs used to make a clinical diagnosis of RPE. RPE is a form of acute lung injury following rapid re-inflation of a collapsed lung and occurs more commonly within $1 \mathrm{~h}$ (64\%) after reexpansion or within $24 \mathrm{~h}$ unexpectedly [6]. Clinical features of RPE in anesthetized patients may include hypoxemia, hypotension, tachycardia, and a pink frothy sputum via an ET tube [7]. However, our patient did not experience hypoxemia, with a $\mathrm{FIO}_{2}$ of 0.5 , and the color of the fluid from the ET tube was similar to that of the fluid drained through the pigtail catheter rather than pink frothy sputum. Thus, we suspected an APF, which was confirmed by a direct vision of an air leak through an opening of the diaphragm. A chest radiograph may be useful to make a diagnosis; however, it was not taken because it seemed that an anteroposterior chest radiograph in a supine position would not have provided useful information and might have been an infectious source in our immunocompromised patient.

Whereas a bronchopleural fistula requires surgical or bronchoscopic intervention, APF almost does not require surgical intervention. However, APF can be problematic during the relatively long operative time, such as OLT. Perioperative respiratory failure has a major influence on recipient and graft outcome [8], and persistent aspiration of pleural effusion from the lung could worsen the patient's oxygenation and ventilation. Prompt bronchial drainage of aspirated fluid, a lung isolation strategy, and intraoperative surgical repair of an APF was necessary to manage our patient during the remaining operation period. Thus, we changed the standard ET tube to a DLT, and the surgeon attempted to repair the defect on the exposed lung surface via a diaphragmatic opening. Although the defect could not be repaired completely, the fluid drainage through DLT was reduced dramatically by chest tube insertion and patient's oxygenation and ventilation were maintained well thereafter.

In conclusion, the present case showed massive endotracheal transudate due to hydrothorax superimposed to APF, which was treated with prompt bronchial suction of aspirated fluid, lung isolation strategy, and surgical repair of APF to maintain oxygenation and ventilation during OLT.

\section{ORCID}

Jae Hee Woo, http://orcid.org/0000-0002-1993-1687

Hee Jung Baik, http://orcid.org/0000-0002-8877-0274

Youn Jin Kim, http://orcid.org/0000-0001-9189-5839

\section{References}

1. Strauss RM, Boyer TD. Hepatic hydrothorax. Semin Liver Dis 1997; 17: 227-32.

2. Cárdenas A, Arroyo V. Management of ascites and hepatic hydrothorax. Best Pract Res Clin Gastroenterol 2007; 21: 55-75.

3. Ginès P, Cárdenas A, Arroyo V, Rodés J. Management of cirrhosis and ascites. N Engl J Med 2004; 350: 1646-54.

4. Roussos A, Philippou N, Mantzaris GJ, Gourgouliannis KI. Hepatic hydrothorax: pathophysiology diagnosis and management. J Gastroenterol Hepatol 2007; 22: 1388-93.

5. Kirsch CM, Chui DW, Yenokida GG, Jensen WA, Bascom PB. Case report: hepatic hydrothorax without ascites. Am J Med Sci 1991; 302: 103-6.

6. Mahfood S, Hix WR, Aaron BL, Blaes P, Watson DC. Reexpansion pulmonary edema. Ann Thorac Surg 1988; 45: 340-5.

7. Neustein SM. Reexpansion pulmonary edema. J Cardiothorac Vasc Anesth 2007; 21: 887-91.

8. Huang CT, Lin HC, Chang SC, Lee WC. Pre-operative risk factors predict post-operative respiratory failure after liver transplantation. PLoS One 2011; 6: e22689. 